\title{
NÍVEL DE PROTEÇÃO TÉRMICA DA CASCA DE QUATRO ESPÉCIES LENHOSAS E A RELAÇÃO DA ARQUITETURA DA CASCA COM A TRANSFERÊNCIA DE CALOR
}

\author{
BARK THERMAL PROTECTION LEVEL OF FOUR TREE SPECIES AND THE RELATIONSHIP \\ BETWEEN BARK ARCHITECTURE AND HEAT TRANSFER
}

\author{
Ailton Teixeira do Vale ${ }^{1}$ Priscila Salomão Elias²
}

\begin{abstract}
RESUMO
A casca é um tecido protetor do câmbio e a exposição a temperaturas acima de $60^{\circ} \mathrm{C}$ durante 1 a 2 minutos é um dos principais fatores que causam a mortalidade de árvores em incêndios florestais. Neste trabalho foi estudado o nível de proteção da casca, como isolante térmico, de três espécies do cerrado: Pterodon pubescens, Vochysia thyrsoidea e Sclerolobium paniculatum e do Eucalyptus grandis e a influência da arquitetura da casca na transferência de calor. Foram retiradas amostras na forma de painéis da casca de cinco árvores de cada espécie. Determinou-se o tempo necessário para que a temperatura no câmbio alcançasse $60^{\circ} \mathrm{C}$ (temperatura letal) e o coeficiente de rugosidade (arquitetura) pela relação entre a área real e a área nominal da amostra. O Sclerolobium paniculatum, com menor espessura de casca, possui o menor tempo de resistência ao calor e o Eucalyptus grandis e o Pterodon pubescens os maiores tempos de resistência ao calor. A exceção foi a gomeira que com a maior espessura de casca possui um dos menores tempos de resistência ao calor. A espécie com maior correlação entre o tempo de resistência ao calor e a espessura de casca total foi o Sclerolobium paniculatum $(\mathrm{r}=0,93)$. O Pterodon pubescens e o Eucalyptus grandis possuem $r=0,73$ e $r=0,56$, respectivamente, e a gomeira possui um baixo coeficiente de correlação $(r=$ $0,34)$. A rugosidade da gomeira foi maior e diferiu significativamente das demais. No entanto, o coeficiente de correlação entre o tempo e a rugosidade não foi significativo para todas as espécies, sugerindo que o aumento da rugosidade não influenciou a transferência de calor através da casca.
\end{abstract}

Palavras-chave: transferência de calor; arquitetura e casca.

\section{ABSTRACT}

The bark is a protective tissue of the tree-cambium and the exposition to temperatures above $60^{\circ} \mathrm{C}$ for 1 to 2 minutes, which is one of the main factors that cause tree mortality in forest fires. In this research, it was studied the bark protection levels of Eucalyptus grandis and of three tree-species of 'cerrado': Pterodon pubescens, Sclerolobium paniculatum and Vochysia thyrsoidea and the effects of these tree bark architectures in heat transfer. Samples were taken from bark panels representing five trees of each species. The time of heat exposure required to damage the tree-cambium until temperature reached $60^{\circ} \mathrm{C}$ (lethal temperature) and the roughness coefficient (architecture) was estimated by the ratio between the actual area and the nominal sample area. The Sclerolobium paniculatum, with thinner bark, showed the shortest time and Eucalyptus grandis and Pterodon pubescens the longer time. The only exception was Vochysia thyrsoidea with a greater thickness and showed the shortest times. The tree species with the highest correlation between time and the total thickness was Sclerolobium paniculatum $(\mathrm{r}=0,93)$. Pterodon pubescens and Eucalyptus grandis showed $\mathrm{r}=0,73$ and $\mathrm{r}=0,56$, respectively and Vochysia thyrsoidea presented the lower correlation coefficient $(0,34)$. The highest roughness was observed for Vochysia thyrsoidea, which was significantly different from the other tree-species. However, the correlation coefficient between time and surface roughness was not significant for all tree-species, which suggest that the

1 Engenheiro Florestal, Dr., Professor Associado II da Universidade de Brasília, Campus Universitário Darcy Ribeiro, Asa Norte, CEP 70910-900, Brasília (DF), Brasil. ailton.vale@gmail.com

2 Engenheira Florestal, Técnico do Núcleo Rural Boa Esperança II, Chácara Portal do Sol, Casa 6, Lago Norte, CEP 71507-991, Brasília (DF), Brasil. priscila_salomao@hotmail.com

Recebido para publicação em 25/10/2011 e aceito em 2/06/2013 
increase of the roughness did not affect the heat transfer through the bark.

Keywords: heat transfer; tree architecture and bark.

\section{INTRODUÇÃO}

O Cerrado é uma formação vegetal complexa que está entre as cinco maiores vegetações do Brasil e é classificado como savana tropical. Os principais fatores que condicionam as savanas tropicais são: clima, com uma estação seca bem definida e outra chuvosa; solos com diferentes níveis de umidade, mas geralmente ácidos e pobres em nutrientes; fogo; utilização da vegetação por herbívoros; geologia; geomorfologia e o histórico de cada local (MONTGOMERY e ASKEW, 1983; COLE, 1986). A combinação desses fatores em cada local proporciona uma grande variação na paisagem natural (EITEN, 1972; FROST et al., 1987; RIBEIRO e WALTER, 1998). O Cerrado é composto por diversas fisionomias, sendo que o cerrado sensu stricto se refere à comunidade mais comum na sua paisagem e mais amplamente distribuída no Distrito Federal (SEMARH, 2004).

As queimadas no cerrado, de origem natural ou antrópica, têm ocorrido por milhares de anos e são comuns durante a estação seca, quando a redução de umidade da vegetação aumenta a sua capacidade de combustão (COUTINHO, 1990; VICENTINI, 1992; MIRANDA et al., 1996). O estudo dos efeitos do fogo sobre os componentes do ambiente é dificultado pelo fato do fogo ser um elemento complexo, que pode possuir características diversas e consequentemente efeitos diversos. As variações das condições do ambiente e do combustível fazem com que cada queimada possua um comportamento característico (GUEDES, 1993). O manejo do ecossistema com o uso do fogo é uma ferramenta útil, que ajuda a controlar a quantidade de combustível, diminuindo assim o risco de queimadas acidentais desastrosas (SCOTT, 1984). Para controlar e prever melhor os prejuízos causados por queimadas controladas ou acidentais é essencial conhecer os limites de tolerância dos vegetais às altas temperaturas (VINES, 1968).

Algumas espécies que vivem em ambientes propícios ao fogo possuem mecanismos que permitem a sua sobrevivência. Soares e Batista (2007) apontaram a casca, por ser um excelente isolante térmico, como o mecanismo mais importante da árvore na proteção contra incêndios florestais. No cerrado existem vários tipos de cascas, que vão da casca fina à espessa e da lisa à fendilhada, passando por todas as suas formas intermediárias (PEREIRA et al., 1993). Para prever o impacto do fogo sobre a vegetação é essencial o desenvolvimento de estudos sobre cascas de árvores (SPALT e REIFSNYDER, 1962; DAVIS, 1959). A casca funciona como um tecido protetor do câmbio que é responsável pelo crescimento secundário do vegetal (ESAU, 1977). A morte do câmbio, quando submetido a altas temperaturas é um dos principais fatores que causam a mortalidade de árvores em incêndios florestais (BYRAM, 1958). De uma forma geral, considera-se que a temperatura capaz de causar a morte de células e tecidos é de $60^{\circ} \mathrm{C}$, por um tempo de 1 a 2 minutos (BYRAM, 1958; HARE, 1961; KAYLL, 1963; UHL e KAUFFMAN, 1990). A morte do câmbio está relacionada às temperaturas que ocorrem durante um incêndio e ao tempo de exposição da árvore, pois uma temperatura só será letal se for mantida por um tempo adequado (WRIGHT e BAILEY, 1982). Segundo estudos realizados por Kayll (1963), a quantidade de calor que chega ao câmbio é inversamente proporcional à espessura da casca e diretamente proporcional a sua umidade. Vale et al. (2006) concluíram que a porção morta da casca tem maior influência na qualidade isolante da mesma, em comparação com a parte viva. Existem ainda poucos estudos a respeito da influência da arquitetura ou área superficial da casca na transferência de calor até o câmbio.

Com relação à variação da temperatura ambiente durante incêndios no Cerrado, Miranda et al. (1993) observaram que, a diferentes alturas, as temperaturas máximas do ar variaram de $85^{\circ} \mathrm{C}$ a $840^{\circ} \mathrm{C}$ durante queimadas em três diferentes fisionomias do cerrado, incluindo o cerrado sensu stricto. A maioria das máximas temperaturas do ar neste estudo foi registrada a $60 \mathrm{~cm}$ de altura. Conforme Frost e Robertson (1985) há uma variação de $200^{\circ} \mathrm{C}$ a $800^{\circ} \mathrm{C}$ nas temperaturas do ar registradas durante incêndios em savanas. $\mathrm{O}$ fogo na Fazenda Água Limpa ocorre, em geral, a cada cinco anos, e registros indicam que a temperatura média do ar a $60 \mathrm{~cm}$ de altura durante os incêndios é de $600^{\circ} \mathrm{C}$ (FIEDLER et al., 2004).

O presente trabalho teve como objetivos investigar o nível de proteção da casca, como isolante térmico, de três espécies do cerrado: 
Pterodon pubescens (sucupira branca); Vochysia thyrsoidea (gomeira); Sclerolobium paniculatum (carvoeiro) e do Eucalyptus grandis (eucalipto) e a influência da arquitetura da casca na transferência de calor.

\section{MATERIAL E MÉTODOS}

\section{Coleta do material}

O experimento foi realizado na estação experimental Fazenda Água Limpa (FAL) da Universidade de Brasília (UnB), localizada a uma altitude de $1100 \mathrm{~m}$ nas coordenadas $15^{\circ} 56^{\prime}-15^{\circ} 59^{\prime} \mathrm{S}$ e $47^{\circ} 55^{\prime}-47^{\circ} 58^{\prime}$ WGr.

Para a realização deste experimento foram escolhidas ao acaso cinco árvores de cada uma das seguintes espécies: Vochysia thyrsoidea, Sclerolobium paniculatum, Pterodon pubescens e Eucalyptus grandis; sendo que, nestas, o diâmetro a altura do peito (DAP) foi acima de $15 \mathrm{~cm}$, em área de vegetação do tipo cerrado sensu stricto e em área de plantação de Eucalyptus grandis na FAL. Com auxílio de ferramentas de corte, foram retiradas até três amostras (Figura 1) da casca de cada árvore a 40,80 e $130 \mathrm{~cm}$ de altura, na forma de painéis de $9,5 \times 11 \mathrm{~cm}$. Para proteger a planta contra pragas e doenças, foi aplicada a calda bordalesa nas áreas que ficaram expostas na árvore, devido à retirada das amostras. Imediatamente após a coleta, as amostras foram identificadas, colocadas em sacos plásticos

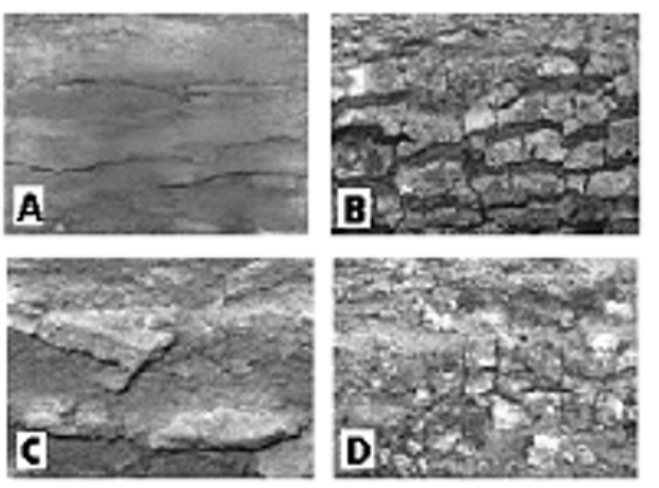

FIGURA 1: Amostras de cascas: A: Eucalyptus grandis, B: Sclerolobium paniculatum, C: Vochysia thyrsoidea, D: Pterodon pubescens).

FIGURE 1: Sample barks: A: Eucalyptus grandis, B: Sclerolobium paniculatum, C: Vochysia thyrsoidea, D: Pterodon pubescens. que foram hermeticamente fechados e levados para o laboratório de Propriedades Energéticas da UnB, localizado na FAL.

O painel de 9,5 x $11 \mathrm{~cm}$ foi utilizado para determinação: da espessura, da proporção de casca viva e morta, da área superficial real e nominal da casca e do tempo necessário para que a temperatura do câmbio alcançasse $60^{\circ} \mathrm{C}$ (temperatura letal).

\section{Determinação das dimensões e do coeficiente de rugosidade da amostra}

O valor médio da espessura total (casca viva mais casca morta) e da espessura da casca viva da amostra foi obtido a partir de quatro determinações realizadas com um paquímetro digital. Para a Vochysia thyrsoidea, que possui cristas e vales muito pronunciados, a espessura total foi tomada a partir do ponto mais alto da crista da parte morta. A casca morta foi determinada pela diferença entre a espessura total da casca e a espessura da casca viva.

A área nominal (An) da superfície da amostra foi obtida, com uma régua graduada, pelo produto entre a largura e o comprimento da amostra.

A área real (Ar) da superfície, que inclui a rugosidade da parte morta da casca, foi determinada como segue: a casca foi colocada dentro de um saco de polietileno de $0,05 \mathrm{~mm}$ de espessura; foi realizado um vácuo com auxílio de uma bomba de vácuo, fazendo com que toda a superfície da casca fosse recoberta, com o saco de polietileno acompanhando, o máximo possível, a rugosidade da amostra (Figura 2A); em seguida, com um

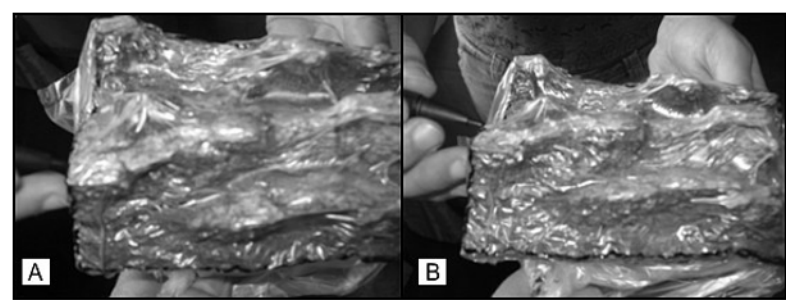

FIGURA 2: A: Posicionamento do saco de polietileno cobrindo a superfície da amostra da casca de Vochysia thyrsoidea e B: Marcação da área superficial real da casca.

FIGURE 2: Positioning of the polyethylene bag covering the surface of the sample bark of Vochysia thyrsoidea and B: Marking the actual surface area of the bark. 
pincel atômico foram demarcados os limites da área superficial (Figura 2B); a casca foi então retirada do saco e com auxílio de uma régua graduada, foram realizadas as medidas da largura e do comprimento da área delimitada pelo desenho no saco plástico, obtendo os valores reais de largura e comprimento da superfície da amostra.

Determinou-se a relação entre "Ar" e "An" $(\mathrm{Cr}=\mathrm{Ar} / \mathrm{An})$, denominando-a de coeficiente de rugosidade $(\mathrm{Cr})$ ou da arquitetura da superfície da amostra.

\section{Determinação do tempo de transferência de calor}

Utilizando-se quatro placas de MDF (Medium Density Fiberboard), coladas em ângulo reto, foi construída uma caixa de $20 \times 18 \mathrm{~cm}$ de seção. A parte interna da caixa foi preenchida com argamassa refratária, formando quatro paredes internas, ocas no centro e contendo três furos para entrada de ar (Figura 3A).

Cada amostra de casca foi posicionada com a parte externa (morta) voltada para baixo em contato direto com a chama produzida por um bico de Bunsen instalado sob a caixa; e a parte interna (viva), contendo o câmbio, voltado para cima em contato com um termômetro, sustentado por uma presilha de madeira (Figura 3B).

Para garantir que a leitura da temperatura no câmbio fosse aquela proveniente da transferência de calor da chama através da casca, uma peça de lã de vidro de $12 \times 14 \mathrm{~cm}$ e $5 \mathrm{~cm}$ de espessura, com um furo de $4 \times 4 \mathrm{~cm}$ para que a chama ficasse em contato direto com a casca, foi colocada sob a casca e outra peça de $9 \times 10 \mathrm{~cm}$ com um furo de $1 \mathrm{~cm}$ de diâmetro no centro para passagem do termômetro, foi posicionada sobre a casca (Figura 3B).
Em seguida, a chama foi acesa iniciando a cronometragem utilizando-se de um cronômetro Technos TEC 965ZF, até que a temperatura do termômetro em contato com o câmbio atingisse $60^{\circ} \mathrm{C}$, encerrando o ensaio.

A temperatura da chama foi monitorada por um termopar de níquel cromoacoplado a um termômetro digital Koban KT 160A, colocado através de um furo sobre a chama. Aleatoriamente foram consideradas duas temperaturas do câmbio $\left(40^{\circ} \mathrm{C}\right.$ e a $\left.60^{\circ} \mathrm{C}\right)$ para registro da temperatura da chama, utilizando a média entre elas. Durante o experimento, a temperatura média da chama na parte externa da casca ficou em média $588^{\circ} \mathrm{C}$.

\section{Análise estatística}

Foram realizadas análise de variância e análise de regressão considerando o tempo, a espessura total da casca e a rugosidade.

\section{RESULTADOS E DISCUSSÃO}

Na Tabela 1 estão os valores médios para o tempo, a espessura e o coeficiente de rugosidade para as cascas de Vochysia thyrsoidea, Sclerolobium paniculatum, Pterodon pubescens e Eucalyptus grandis.

\section{Nível de proteção térmica}

\section{Nível de proteção térmica da casca}

Em geral, o comportamento das cascas quanto ao aumento do tempo de aquecimento até $60^{\circ} \mathrm{C}$, com o aumento da espessura, foi semelhante a resultados encontrados por outros autores, como

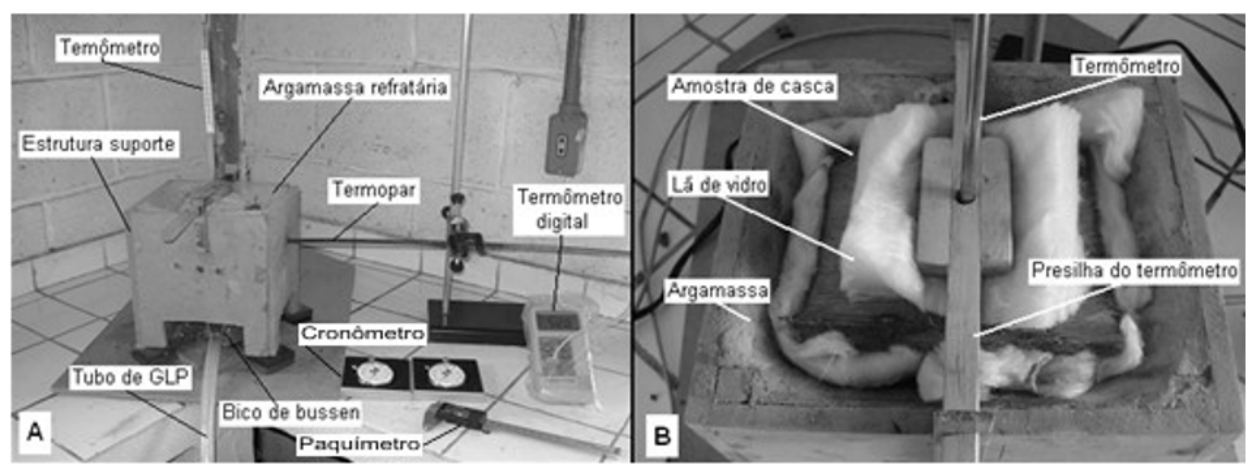

FIGURA 3: A: Vista geral da estrutura suporte e B: Posicionamento da amostra no suporte.

FIGURE 3: A: General view of the support structure and B: Positioning of the sample in the holder. 
TABELA 1: Características da casca de Vochysia thyrsoidea (gomeira), Sclerolobium paniculatum (carvoeiro), Pterodon pubescens (sucupira) e Eucalyptus grandis (eucalipto).

TABLE 1: Characteristics of the bark of Vochysia thyrsoidea (gomeira), Sclerolobium paniculatum (carvoeiro), Pterodon pubescens (sucupira) and Eucalyptus grandis (eucalipto).

\begin{tabular}{cccc}
\hline Espécie & Tempo $(\mathrm{min})$ & Espessura Total $(\mathrm{cm})$ & Coeficiente de rugosidade \\
\hline Eucalipto & 15,76 & 1,650 & 1,11 \\
CV $(\%)$ & $(16,05)$ & $(19,71)$ & $(6,53)$ \\
Carvoeiro & 7,23 & 1,330 & 1,12 \\
CV(\%) & $(25,42)$ & $(21,12)$ & $(14,78)$ \\
Gomeira & 8,21 & 2,200 & 1,48 \\
CV $(\%)$ & $(26,42)$ & $(22,44)$ & $(18,78)$ \\
Sucupira & 14,58 & 1,917 & 1,11 \\
CV $(\%)$ & $(15,71)$ & $(9,52)$ & $(9,23)$ \\
\hline
\end{tabular}

Gava et al. (1995), trabalhando com Eucalyptus tereticornis e Eucalyptus torelliana; Vines (1968), trabalhando com árvores de uma floresta nativa e Pinard e Huffman (1997), trabalhando com árvores de uma floresta nativa no leste da Bolívia. Neste sentido observa-se pelos dados da Tabela 1, que o carvoeiro, espécie com menor espessura total de casca $(1,33 \mathrm{~cm})$ foi o de menor tempo de resistência ao calor $(7,23 \mathrm{~min})$ o eucalipto e a sucupira, espécies com, respectivamente, $1,65 \mathrm{~cm}$ e 1,917 $\mathrm{cm}$ de espessura total de casca possuem os maiores tempos de resistência ao calor $(15,76$ min e 14,58 min, respectivamente) e, portanto, os maiores níveis de proteção do câmbio. A exceção foi a gomeira que com espessura de casca de $2,20 \mathrm{~cm}$ possui tempo de 8,21 minutos de resistência ao calor. A explicação está na arquitetura (rugosidade) da casca morta, que é formada por cristas e vales (Figura 4). No posicionamento da amostra para o ensaio, as cristas e os vales ficam voltados para baixo em contato direto com a chama. A espessura total foi tomada a partir da crista, mas a chama, durante o experimento, além de atingir a crista atingia também os vales. Portanto, deve-se considerar que o calor foi transferido também a partir do vale da casca até o câmbio, diminuindo assim a distância e com isso o tempo de aquecimento.

A arquitetura da casca do eucalipto, do carvoeiro e da sucupira difere muito da casca da gomeira, como pode ser observado na Figura 1. Portanto, não basta considerar a espessura total na relação tempo x espessura; é preciso considerar as reentrâncias (vales) que porventura existam na estrutura.

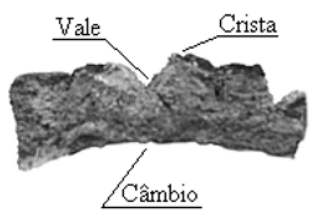

FIGURA 4: Casca de Vochysia thyrsoidea.

FIGURE 4: Bark of Vochysia thyrsoidea.

Na Tabela 2 está o resultado da análise de variância para a espessura total da casca das espécies em estudo. Ao nível de significância de $1 \%$, as médias de espessura total das espécies são significativamente diferentes, neste sentido foi realizado o teste de Tukey para separar as médias, conforme Tabela 3.

TABELA 2: Análise de variância para a espessura total da casca das espécies Eucalyptus grandis (eucalipto), Sclerolobium paniculatum (carvoeiro), Vochysia thyrsoidea (gomeira) e Pterodon pubescens (sucupira).

TABLE 2: Analysis of variance for the total thickness of the bark of the species Eucalyptus grandis (eucalipto), Sclerolobium paniculatum (carvoeiro), Vochysia thyrsoidea (gomeira) and Pterodon pubescens (sucupira).

\begin{tabular}{ccccc}
\hline $\begin{array}{c}\text { Fonte de } \\
\text { variação }\end{array}$ & $\begin{array}{c}\text { Graus de } \\
\text { liberdade }\end{array}$ & $\begin{array}{c}\text { Soma de } \\
\text { Quadrados }\end{array}$ & $\begin{array}{c}\text { Quadrado } \\
\text { Médio }\end{array}$ & F calculado \\
\hline Entre & 3 & 3,2995 & 1,0998 & 9,5008 \\
Dentro & 27 & 3,1255 & 0,1158 & \\
Total & 30 & 6,4250 & & \\
& & &
\end{tabular}


TABELA 3: Comparação entre médias de espessura total de cascas de Eucalyptus grandis, Sclerolobium paniculatum, Vochysia thyrsoidea e Pterodon pubescens, a 5\% de significância, pelo teste de Tukey.

TABLE 3: Comparison of average total thickness of bark of Eucalyptus grandis, Sclerolobium paniculatum, Vochysia thyrsoidea and Pterodon pubescens, the $5 \%$ significance by Tukey test.

\begin{tabular}{cc}
\hline Tratamento & Espessura total da casca \\
\hline Gomeira & $2,200 \mathrm{a}$ \\
Sucupira & $1,917 \mathrm{ab}$ \\
Eucalipto & $1,650 \mathrm{bc}$ \\
Carvoeiro & $1,330 \mathrm{c}$ \\
\hline
\end{tabular}

Médias seguidas de mesma letra não diferem entre si pelo teste Tukey ao nível de 5\% de probabilidade.
A biodiversidade do cerrado é elevada. $\mathrm{O}$ número de plantas vasculares é superior àquele encontrado na maioria das regiões do mundo, com mais de 7000 espécies (MENDONÇA et al., 1998). É importante que sejam realizados mais estudos a respeito do nível de proteção da casca, como isolante térmico, de espécies do cerrado, pois, para muitas dessas espécies, ainda não existem essas informações, as quais podem auxiliar na prevenção e no controle dos prejuízos causados por queimadas e no manejo do ecossistema com o uso do fogo.

A Figura 5 ilustra a dispersão dos dados de tempo em relação à espessura total da casca. A espécie com maior coeficiente de determinação entre a espessura total de casca e o tempo de elevação da temperatura, para atingir $60^{\circ} \mathrm{C}$ no câmbio foi o Sclerolobium paniculatum (carvoeiro) com um $\mathrm{r}=0,92$. O Pterodon pubescens (sucupira) e o Eucalyptus grandis (eucalipto) também possuem correlação positiva entre a espessura total
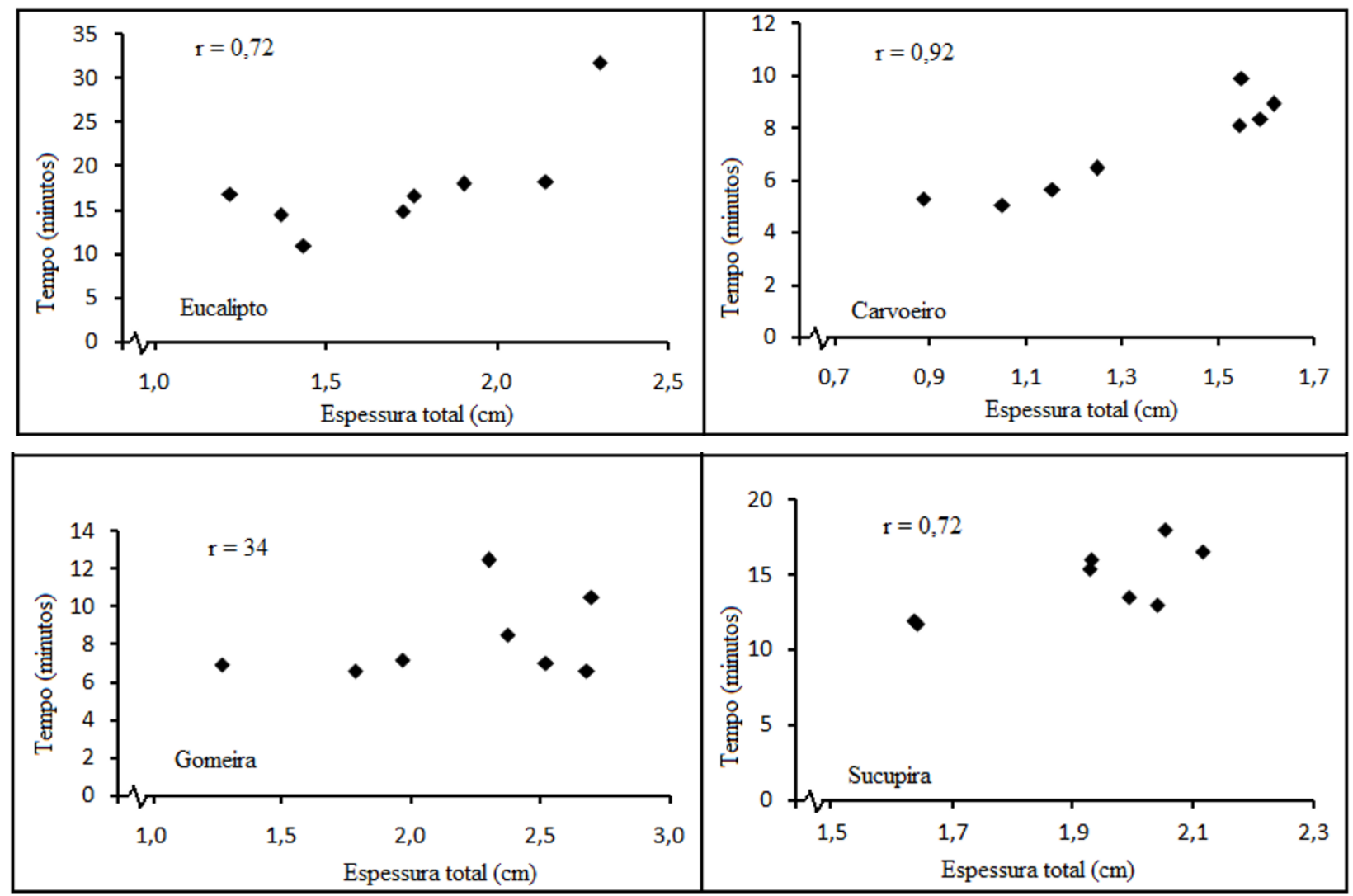

FIGURA 5: Tempo entre o início do aquecimento da casca pela chama até $60^{\circ} \mathrm{C}$ em função da espessura total. A: Eucalyptus grandis (eucalipto), B: Sclerolobium paniculatum (carvoeiro), C: Vochysia thyrsoidea (gomeira) e D: Pterodon pubescens (sucupira).

FIGURE 5: Time between the beginning the heating of the bark by the fire until $60^{\circ} \mathrm{C}$ as a function of thickness. A: Eucalyptus grandis (eucalipto), B: Sclerolobium paniculatum (carvoeiro), C: Vochysia thyrsoidea (gomeira) and D: Pterodon pubescens (sucupira). 
TABELA 4: Análise de variância para o coeficiente de rugosidade das espécies Eucalyptus grandis, Sclerolobium paniculatum, Vochysia thyrsoidea e Pterodon pubescens.

TABLE 4: Analysis of variance for the roughness coefficient of the species Eucalyptus grandis, Sclerolobium paniculatum, Vochysia thyrsoidea and Pterodon pubescens.

\begin{tabular}{ccccc}
\hline $\begin{array}{c}\text { Fonte de } \\
\text { variação }\end{array}$ & $\begin{array}{c}\text { Graus de } \\
\text { Liberdade }\end{array}$ & $\begin{array}{c}\text { Soma de } \\
\text { Quadrado }\end{array}$ & $\begin{array}{c}\text { Quadrado } \\
\text { Médio }\end{array}$ & $\begin{array}{c}\text { F } \\
\text { calculado }\end{array}$ \\
\hline Tratamento & 3 & 0,7853 & 0,2618 & 8,4425 \\
Resíduo & 27 & 0,8372 & 0,0310 & \\
\hline Total & 30 & 1,6226 & & \\
\hline
\end{tabular}

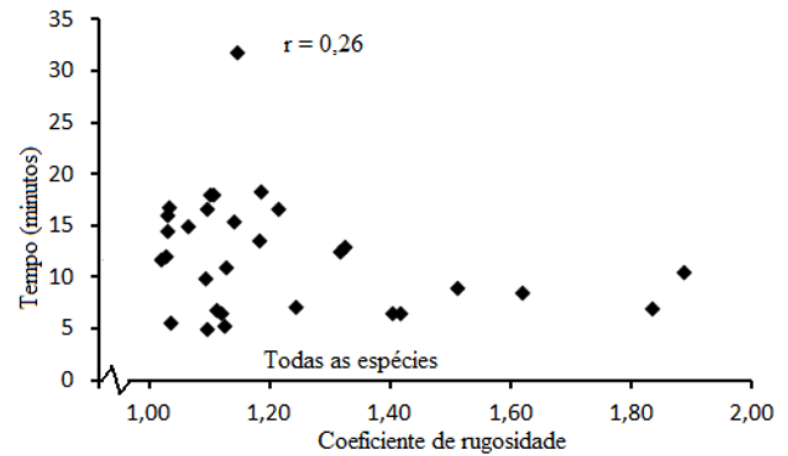

FIGURA 7: Tempo decorrido entre o início do aquecimento da casca pela chama até o alcance de $60^{\circ} \mathrm{C}$ de temperatura do câmbio em função do fator de rugosidade para todas as espécies.

FIGURE 7: Time between the beginning the heating of the bark by the fire until $60^{\circ} \mathrm{C}$ as a function of thickness for all species.
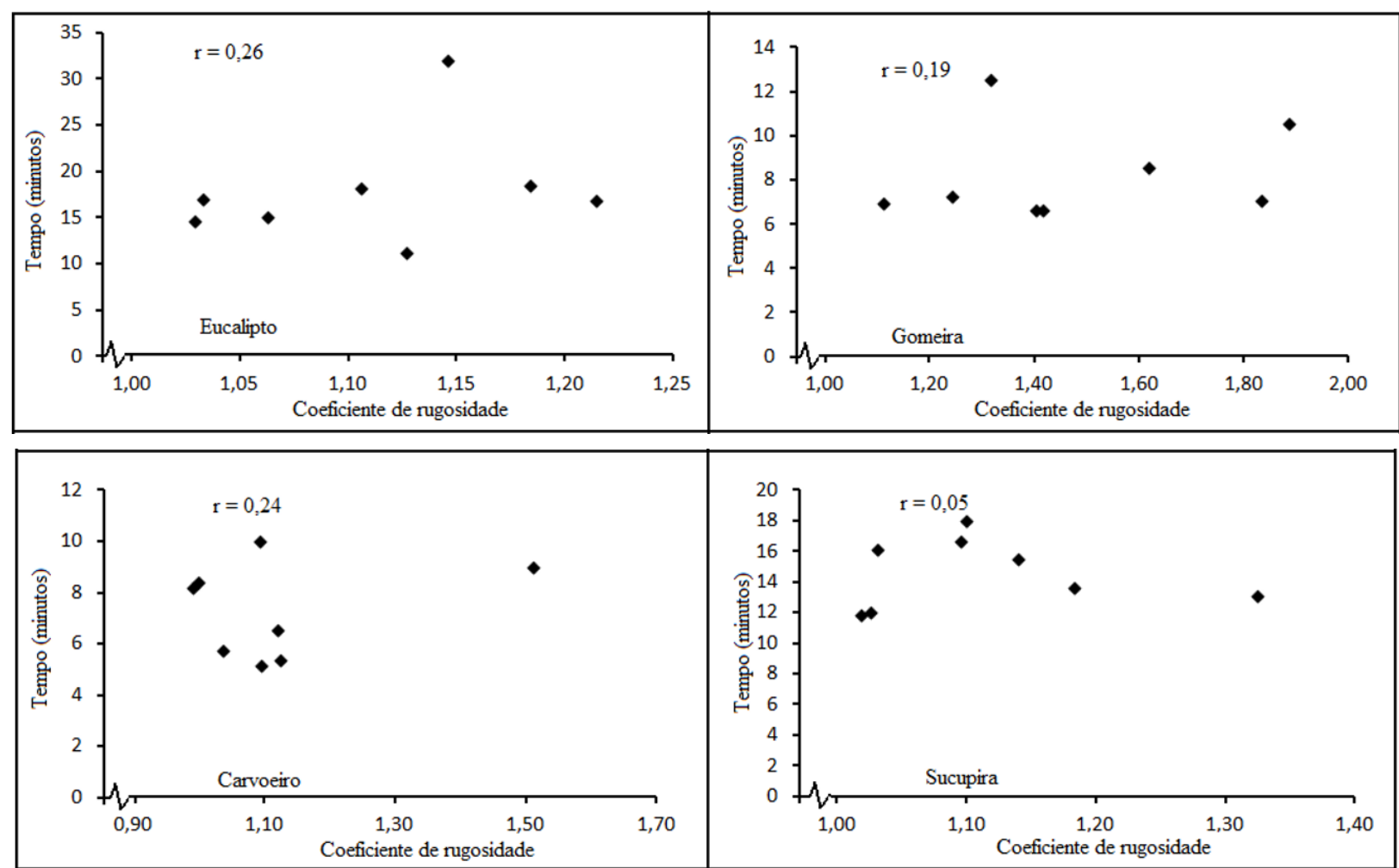

FIGURA 6: Tempo decorrido entre o início do aquecimento da casca pela chama até o alcance de $60^{\circ} \mathrm{C}$ de temperatura do câmbio em função do fator de rugosidade. A: Eucalyptus grandis (eucalipto), B: Sclerolobium paniculatum (carvoeiro), C: Vochysia thyrsoidea (gomeira) e D: Pterodon pubescens (sucupira).

FIGURE 6: Time between the beginning of the heating the bark by the fire until $60^{\circ} \mathrm{C}$ as a function of thickness. A: Eucalyptus grandis (eucalipto), B: Sclerolobium paniculatum (carvoeiro), C: Vochysia thyrsoidea (gomeira) and D: Pterodon pubescens (sucupira). 
de casca e o tempo de elevação da temperatura $(\mathrm{r}=0,72)$. A Vochysia thyrsoidea possui um coeficiente de correlação entre o tempo e a espessura total muito baixo, $(r=0,34)$, pelos motivos expostos anteriormente.

Nivel de proteção térmica e a arquitetura da casca

A gomeira possui casca morta com fissuras e cristas descontínuas e sinuosas; a sucupira possui casca morta áspera, com depressões irregulares de placas que se soltam, fissurada com veios profundos nas árvores mais velhas; o carvoeiro possui casca com estrias transversais (SILVA JÚNIOR, 2005). O eucalipto possui casca morta caracterizada pelo desprendimento de lâminas irregulares.

A arquitetura (rugosidade) da casca foi aqui estabelecida pela relação entre a área real e a área nominal da casca, denominada coeficiente de rugosidade. As médias de rugosidade da casca entre as espécies diferem significativamente ao nível de $1 \%$ de significância, conforme a análise de variância da Tabela 4, sendo que a rugosidade da gomeira é maior e difere das demais, conforme $o$ teste de Tukey (Tabela 5).

TABELA 5: Comparação entre médias de coeficientes de rugosidade das espécies Eucalyptus grandis, Sclerolobium paniculatum, Vochysia thyrsoidea e Pterodon pubescens, a $5 \%$ de significância, pelo teste de Tukey.

TABLE 5: Comparison of average roughness coefficients of the species Eucalyptus grandis, Sclerolobium paniculatum, Vochysia thyrsoidea and Pterodon pubescens, the $5 \%$ significance by Tukey test.

\begin{tabular}{cc}
\hline Tratamento & Coeficiente de rugosidade \\
\hline Gomeira & $1,48 \mathrm{a}$ \\
Carvoeiro & $1,12 \mathrm{~b}$ \\
Sucupira & $1,11 \mathrm{~b}$ \\
Eucalipto & $1,11 \mathrm{~b}$ \\
\hline
\end{tabular}

Médias seguidas de mesma letra não diferem entre si pelo teste Tukey ao nível de $5 \%$ de probabilidade.

As espécies, quando analisadas separadamente, não possuem correlação significativa entre o tempo e o coeficiente de rugosidade (Figura 6). Isso sugere que a rugosidade não interfere na capacidade de proteção térmica da casca, o que pode ser observado pelos baixos coeficientes de correlação $(<0,3$ para todas as espécies). Considerando que a variação da rugosidade e, por conseguinte, da arquitetura da casca variou pouco dentro da espécie, os dados de todas as espécies foram analisados conjuntamente, conforme a Figura 7, que ilustra a área superficial e, consequentemente, a arquitetura da casca não contribuisignificativamenteparaaproteçãodocâmbio nos ensaios de transferência de calor. Isto aconteceu provavelmente porque mais importante do que a arquitetura da casca é a distância entre a fonte de calor e o câmbio.

\section{CONCLUSÕES}

Pelos resultados expostos pode-se concluir que a arquitetura da casca, ou seja, o aumento da área da casca exposta ao fogo não teve influência significativa na proteção da mesma contra o aumento da temperatura, por outro lado, em geral, o aumento da espessura da casca promove maior nível de proteção do câmbio. No entanto, para cascas fissuradas, quando se toma a espessura total a partir das "cristas", a relação entre espessura e tempo de transferência de calor não ocorreu, uma vez que além da transferência de calor a partir da "crista" tem-se também transferência de calor a partir dos "vales".

\section{AGRADECIMENTOS}

Agradecemos ao técnico de laboratório Vandui Francisco de Siqueira Dantas pela ajuda no trabalho de campo e ao programa ProIC/CNPq-Unb pela bolsa concedida.

\section{REFERÊNCIAS BIBLIOGRÁFICAS}

BYRAM, G. M. Some basic thermal processes controlling the effects of fire on living vegetation. USDA Forest Service Southeast Forest Experiment Station, Res. Note, n.114, 1958. 2 p.

COLE, M. The Savannas, biogeography and geobotany. London: Academic press, 1986.

COUTINHO, L. M. Fire in the ecology of the Brazilian Cerrado. In: GOLDAMMER, J. G. (Ed.). Fire in the Tropical Biota - Ecosystem Processes and Global Challenges. Berlin: Springer-Verlag, 
1990. p.82-105.

DAVIS, K. P. Forest fire: control and use. New York: John Wiley \& Sons, 1959.

EITEN, G. The cerrado vegetation of Brazil. The Botanical Review, v. 2, n. 38, p. 201-341, 1972. ESAU, K. Anatomy of seed plants. $2^{\text {nd }}$ ed. New York: John Wiley \& Sons, 1977. 550 p.

FIEDLER, N. C.K.L. et al. Efeito de incêndios florestais na estrutura e composição florística de uma área de cerrado sensu stricto na fazenda Água Limpa-DF. Revista Árvore, v. 28, n. 1, p. 129-138, 2004.

FROST, P. et al. Responses of savannas to stress and disturbance: a proposal for a collaborative program of research. Biology International. Special Issue. Paris, n.10, p.1-82, 1986.

FROST, P. G. H.; ROBERTSON, F. The ecological effects of fires in savannas. In: WALKER, B. H. (Ed.). Determinants of tropical savannas. Oxford: IRL Press Limited, 1987, p.93-140.

GAVA, J. L. et al. Influência da espessura da casca de Eucalytpus torelliana e Eucalyptus tereticornis sobre a variação da temperatura do câmbio durante a ocorrência de um incêndio florestal. IPEF $\mathrm{n}$. 48/49, p. 126-132, 1995.

GUEDES, D. M. Resistência das árvores do cerrado ao fogo: papel da casca como isolante térmico. 1993. 113 f. Dissertação (Mestrado em Ecologia) - Universidade de Brasília, Brasília-DF, 1993.

HARE, R. C. Heat effects on living plants. USDA Forest Service Southeast Forest Experiment Station, Occasional Paper, n.183, 1961. 32 p.

KAYLL, A. J. A technique for studying the fire tolerance of living trunks. Canada: Department of Forestry, , 1963. 22 p.

MENDONÇA, R. et al. Flora vascular do Cerrado. In: SANO S. M.; ALMEIDA S. P. (Eds.). Cerrado: ambiente e flora. Planaltina: EMBRAPA-CPAC, 1998. p. 288-556.

MIRANDA, A. C. et al. Soil and air temperatures during prescribed Cerrado fires in Central Brasil. Journal of Tropical Ecology, v. 9, p. 313-320, 1993.

MIRANDA, H. S. et al. Comportamento do fogo em queimadas de campo sujo. In: MIRANDA, H. S. et. al. (Orgs.). Impactos de Queimadas em Áreas de Cerrado e Restinga. Brasília: ECL/Universidade de Brasília, 1996. p. 1-10.

MONTGOMERY, R. F.; ASKEW, G. P. Soils of tropical savannas. In: BOURLIÉRE, F. (Ed.). Tropical savannas. Amsterdam: Elsevier, 1983. p.63-78.

PEREIRA, B. A. S.; GUEDES, D. M.; VIANNA Jr. Correlação entre espessura da casca e diâmetro do tronco nas espécies do Cerrado. In: CONGRESSO BRASILEIRO DE BOTÂNICA, 1993, São Luiz. Anais... São Luiz, 1993.

PINARD, M. A.; HUFFMAN, J. Fire resistance and bark properties of trees in a seasonally dry forest in eastern Bolivia. Journal of Tropical Ecology, v. 13, n. 5, p. 727-740, 1997.

RIBEIRO, J. F.; WALTER, B. M. T. Fitofisionomias do bioma Cerrado. In: SANO S. M.; ALMEIDA S. P. (Eds.). Cerrado: ambiente e flora. Planaltina: EMBRAPA-CPAC, 1998. p.89-169.

SCOTT, J. D. An historical review of research on fire in South Africa. In: BOOYSEN, P. V.; TAINTON, N. M. (Eds.). Ecological effects of fire in South African ecosystems. Berlim: Springer-Verlag, 1984. p.53-65.

SEMARH-Secretaria de Meio Ambiente e Recursos Hídricos do DF. Caderno técnico: prevenção e combate aos incêndios florestais em Unidades de Conservação. Brasília: Athalaia, 2004. 96 p.

SILVA JÚNIOR, M. C. 100 árvores do cerrado guia de campo. 1. ed. Brasília: Rede de Sementes do Cerrado, v.1, 2005. 278 p.

SOARES, R. V.; BATISTA, A. C. Incêndios Florestais: controle, efeitos e uso do fogo. Curitiba: Ronaldo Viana Soares e Antonio Carlos Batista, 2007. $264 \mathrm{p}$

SPALT, K. W.; REIFSNYDER, W. E. Bark characteristics and fire resistance: a literature survey. USDA Forest Service Southeast Forest Experiment Station, Occasional Paper, n.193, 1962. $19 \mathrm{p}$.

UHL, C.; KAUfFMAN, J. B. Deforestation, fire susceptibility, and potential tree responses to fire in the earstern Amazon. Ecology, v. 71, n. 2, p. 437499, 1990.

VALE, A. T. et al. Influência das propriedades da casca de Vochysia thyrsoidea na transferência de calor para o câmbio. Revista Científica Eletrônica de Engenharia Florestal, n. 13, 2006.

VICENTINI, K. R. C. F. Análise Palinológica de uma Vereda em Cromínia-GO. 1992. 137 f. Dissertação (Mestrado em Ecologia) - Universidade de Brasília, Brasília, 1992.

VINES, R. G. Heat transfer through bark, and the resistance of trees to fire. Australian Journal of Botany, v.16, p.499-514, 1968.

WRIGHT, S. J. ; BAILEY, A.W. Fire Ecology. New York: John Wiley \& Sons. 1982. 501 p. 